\title{
Upper Limb Load as a Function of Repetitive Task Parameters: Part 2-An Experimental Study
}

\author{
Danuta Roman-Liu \\ Tomasz Tokarski
}

Central Institute for Labour Protection - National Research Institute, Warsaw, Poland

The aim of the study was to compare the theoretical indicator of upper limb load with the physiological indicator of musculoskeletal load, which is present while performing a repetitive task (a normalized electromyography [EMG] amplitude recorded from the muscles of the upper limb involved in the performed task). In an experimental study of a repetitive task, the EMG signal from 5 main muscles of the shoulder girdle, arm and forearm was registered: extensor carpi radialis longus, flexor carpi ulnaris, deltoideus anterior, biceps brachii caput breve and trapezius descendent.

The results of the study showed a strong correlation between the theoretical indicator (Integrated Cycle Load) and the physiological indicator (root mean square of a normalized EMG amplitude from the 5 muscles). This proves that the developed theoretical indicator can be accepted as an indicator of upper limb musculoskeletal load during a work task.

electromyography upper limb handgrip force cycle load

\section{INTRODUCTION}

It is important to study the relation between the external load imposed by the work environment and the internal load on muscles. Various indicators of musculoskeletal load which results from a performed task were considered in experimental studies. The most frequent were electromyography (EMG) parameters [1, 2, 3, 4, $5,6,7]$, but also heart rate $[3,6]$, blood pressure $[2,4]$ and subjective measures $[6,8,9]$. EMG is a good tool for the assessment of internal muscle load. Several studies proved that the force of a muscle is proportional to an EMG amplitude $[10,11,12,13,14]$. All those indicators of musculoskeletal load relate to given work conditions described by repetitive task parameters and they can evaluate musculoskeletal load for those conditions only. This means that each work condition must be assessed experimentally, which makes assessment time consuming and expensive. There is a need for an indicator of load which would be based on the parameters describing the performed task. Such an indicator would make it possible to assess musculoskeletal load on the basis of the values of task parameters without the necessity to conduct an experiment. Thus it would be very useful in designing workplaces.

A theoretical indicator of upper limb musculoskeletal load based on repetitive task parameters was developed in a study presented in Part 1 [15]. It is hypothesized that the indicator based on task parameters correlates with a physiological indicator of musculoskeletal load, a normalized EMG amplitude recorded from the muscles of the upper limb involved in performing the task.

\footnotetext{
This study is part of the National Strategic Programme "Occupational Safety and Health Protection in the Working Environment", supported in 1998-2001 by the State Committee for Scientific Research in Poland. The Central Institute for Labour Protection is the Programme's main co-ordinator.

Correspondence and requests for offprints should be sent to Danuta Roman-Liu, Central Institute for Labour Protection - National Research Institute, ul. Czerniakowska 16,00-701 Warszawa, Poland. E-mail: <daliu@ ciop.pl>.
} 
To prove this hypothesis, an experimental study of repetitive tasks was performed. In that study different variants were characterized by different values of repetitive task parameters. The study was performed for variants of load for which the theoretical indicator of musculoskeletal load (Integrated Cycle Load, ICL)—expressing upper limb load—had been calculated. Upper limb load was also assessed on the basis of the physiological indicator of musculoskeletal load based on EMG measurements of the five main upper limb muscles. Verification of the established theoretical indicator of upper limb musculoskeletal load (ICL) was performed by comparing theoretical results $(I C L)$ and experimental ones (root mean square of a normalized EMG amplitude for the registered five muscles).

\section{METHODOLOGY}

\subsection{Participants}

Nine young men, right-hand dominant, aged from 20 to 24 years (average age 21.9 years), were recruited from a group of male students of the Academy of Physical Education in Warsaw, Poland. The participants were healthy, had no history of muscle pain and had similar anthropometric dimensions: their average body mass was $88 \mathrm{~kg}$ (from 80 to 93), body height 179 $\mathrm{cm}$ (from 177 to 182) (Table 1).

The study was carried out with the approval of the Academy of Physical Education Ethics Committee. Before the experiments, an informed

TABLE 1. Characteristics of Participants

\begin{tabular}{lccc}
\hline Participant & $\begin{array}{c}\text { Age } \\
\text { (years) }\end{array}$ & $\begin{array}{c}\text { Body Height } \\
\text { (cm) }\end{array}$ & $\begin{array}{c}\text { Body Mass } \\
\text { (kg) }\end{array}$ \\
\hline MC & 20 & 179 & 77 \\
MG & 22 & 179 & 82 \\
MJ & 21 & 178 & 82 \\
ML & 22 & 177 & 80 \\
MM & 22 & 179 & 81 \\
PG & 23 & 178 & 67 \\
RH & 24 & 182 & 93 \\
RL & 23 & 181 & 78 \\
TB & 20 & 178 & 88 \\
$M$ & 21.89 & 179 & 80 \\
\hline
\end{tabular}

consent was obtained. The participants were financially compensated for their participation.

\subsection{Experimental Layout}

Experiments were performed in 12 variants of external load. During the main experiment, a participant performed a repetitive task in an appropriate variant described by the level of the relative force and duration of each of the four periods of the cycle.

Two types of upper limb strength activities were included. They were handgrip force and the force of supporting the upper limb in a determined posture. The duration and external load in each variant of load was presented in detail in Part 1 [15].

Handgrip force and the EMG signal from five muscles were registered during the experiments. Participants performed the task in a sitting position, with their back straight and the left upper limb relaxed (Figure 1).

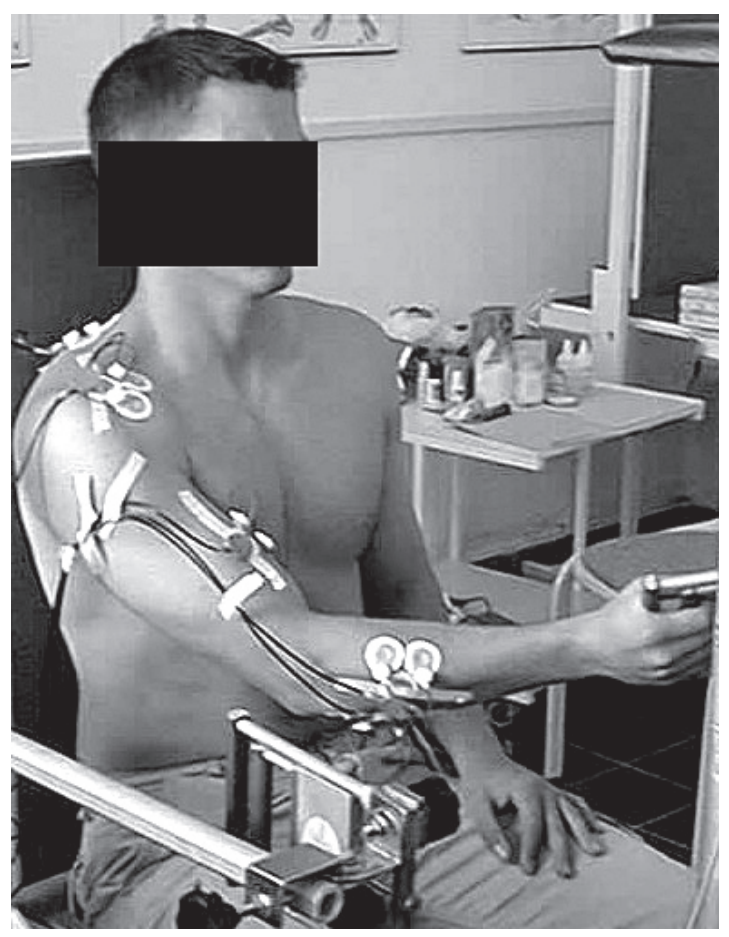

Figure 1. The experimental setup for Boundary Upper Limb Postures A and B.

The sequence of variants was randomized for each participant. During the experiments handgrip force exertion in period 1 and period 3 was imposed by a 5 -s sound signal. Keeping the 
force level demanded sight control of the force. It was accomplished by presenting the value of the force on a screen. The participant's task was to keep handgrip force at a set level.

\subsection{Force Measurements}

Handgrip force was measured with a hand dynamometer against which the participants exerted contractions of muscles in static (isometric) tests. The dynamometer was fastened onto a metal support in a position which imposed on the participants exerting handgrip force in a defined upper limb posture. During the test the participants controlled the level of force with a digital meter.

Measurements of grip strength were performed using equipment which consisted of a DR-3 hand dynamometer with a regulated grip span connected through a WT8-RS 8channel amplifier with a 12-bit analogue-digital converter to a PC-class computer. The equipment was produced by JBA, Poland. The nominal measurement range of the hand dynamometer was $1,200 \mathrm{~N}$ with the maximal linear error lower than 5\%. The specially developed CPS_v_2.0 software supplied additionally made it possible to register and graphically present the actual value of strength from the dynamometer. After the test it was possible to see the measured value.

\subsection{EMG Measurements}

\subsubsection{Procedure and equipment}

The involvement of upper limb muscles in force exertion varies depending on the type of force that is exerted. Activities like lifting, pushing, pulling, pronation and supination-or activities in which force is exerted by the muscles of the hand and forearm only-involve muscles of the whole upper limb. In the experiments, handgrip force was exerted (mostly muscles of the forearm were involved) as well as the force of supporting the upper limb in a determined posture (involving the activity of arm and shoulder muscles).

The following muscles were examined: two muscles of the forearm (extensor carpi radialis longus and flexor carpi ulnaris), two muscles of the arm (deltoideus anterior and biceps brachii caput breve) and one muscle of the shoulder girdle (trapezius descendent).

Those muscles were selected because they represent the component forces exerted during the experiment, i.e., handgrip force and the weight of the upper limb reflected by the lifting force. Those muscles are comparably big and located just under the skin making surface EMG measurement relatively convenient. The anatomical localization of the muscles was done by feel as participants put them to isometric tension.

A Muscle Tester MESPEC 4000 (Mega Electronics, Finland) and an IBM computer were used to measure and store data. The EMG signal was registered though MS-OOS (Medicotes, Denmark) surface electrodes. To ensure consistent electrode placement for each of the test sessions, the participant's skin was marked around the electrodes. The skin was properly prepared to obtain skin resistance below $2 \mathrm{k} \Omega$. Preamplifiers mounted to the electrodes made it possible to register the non-artefacted signal. The EMG signal was sampled through a 12-bit A/D converter with a sampling rate of $1000 \mathrm{~Hz}$.

\subsubsection{Measurements of maximum voluntary contraction of the examined muscles}

Before the experiment, tests for maximum tension for each of the examined muscles were performed. For each of the analyzed muscles maximum force occurs in different activities and directions of the exerted force [16, 17]. Therefore four different tests were performed; for forearm muscles-extensor carpi radialis and flexor carpi ulnaris - tests of maximum muscle tension were the same (maximum handgrip force).

During the tests the participants were asked to build up the force gradually without jerking and to hold the exertion for $3 \mathrm{~s}$. Verbal encouragements were offered during the test. The aim of those measurements was to obtain a maximum amplitude of the EMG signal for normalization for each of the analyzed muscles.

Test 1-maximum tension in muscle trapezius pars descendens. A calibration platform equipped with two adjustable slings was 
used to measure the maximum EMG signal from trapezius pars descendent. The participant stood on a platform in a neutral position with both arms hanging down. He pulled sling handles. In that body position, values of all seven angles defining the upper limb posture were equal to $0^{\circ}$. The procedure had to be performed by pulling the handle smoothly and by using the shoulders (without flexion in the elbow joint).

Test 2-maximum tension in muscle deltoideus anterior. The same platform was used to exert maximum force for muscle deltoideus pars anterior. The participant was in a sitting position, arms abducted at $45^{\circ}$, flexed in the elbow at $90^{\circ}$. Slings were fastened to the elbow. That upper limb location was defined according to the LIMB model [15] as follows: $q_{1}=45^{\circ} ; q_{2}=0^{\circ} ; q_{3}=0^{\circ}$; $q_{4}=90^{\circ} ; q_{5}=0^{\circ} ; q_{6}=0^{\circ} ; q_{7}=0^{\circ}$. The participant exerted the force by lifting his arms vertically.

Test 3-maximum tension in muscle biceps brachii. Maximum activity of muscle biceps brachii was registered during maximum pulling in the upper limb flexed in the arm at $90^{\circ}$ and flexed in the elbow at $90^{\circ}$. The upper limb location was defined according to the LIMB model as follows: $q_{1}=0^{\circ} ; q_{2}=90^{\circ} ; q_{3}=0^{\circ} ; q_{4}=90^{\circ} ; q_{5}=0^{\circ} ; q_{6}=0^{\circ}$; $q_{7}=0^{\circ}$.
Test 4-maximum tension in muscles extensor carpi radialis and flexor carpi ulnaris. The maximum signal for extensor carpi radialis and flexor carpi ulnaris muscles was accepted as that measured during maximum handgrip force. When exerting force, the participant was in a sitting position with his back straight and left limb relaxed. Respective EMG signals from the muscles were measured for each of the four examined upper limb postures (A, B, C and D). The maximum force measured during this test was used as a reference value for the relative force of the handgrip in the same upper limb posture.

\section{ANALYSIS}

The EMG signal amplitude registered from upper limb muscles can be an indicator of the differences in musculoskeletal load according to variants of experiments characterized by external load. An example of a raw EMG signal registered during the experiment is presented in Figure 2.

The average EMG amplitude was calculated by full waverectificationfollowed by integrationover $100 \mathrm{~ms}$. The mean value of the average amplitude was calculated over three cycles of the performed

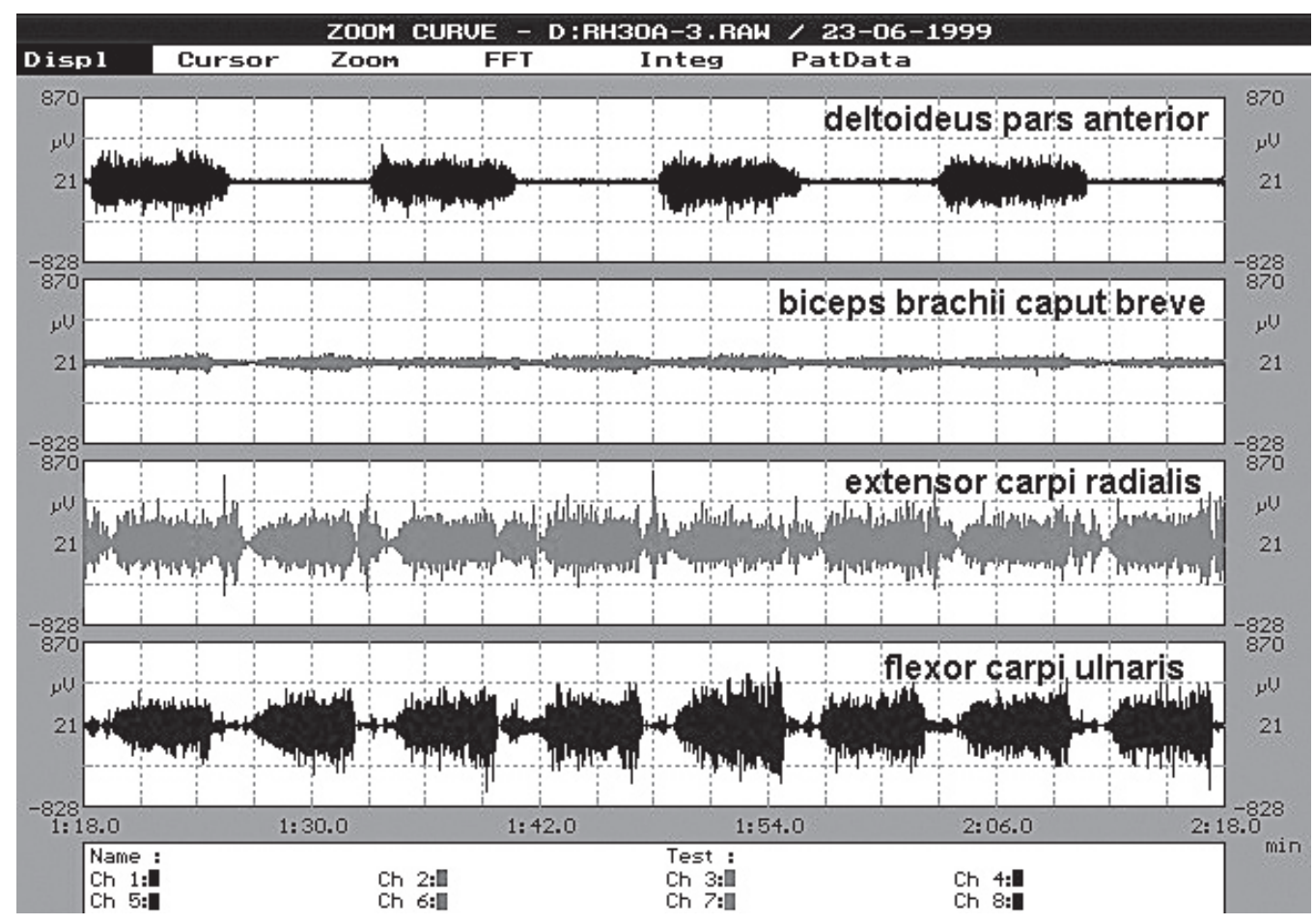

Figure 2. An example of a raw electromyography (EMG) signal registered during the experiment. 
task and normalized. The normalization was performed for each muscle according to values of the amplitude measured during maximum force exertion in a test performed separately for each of the examined muscle before the experiments. Normalization was performed according to a procedure described in Mirka [18]. The parameter calculated in this way (NEMG) was considered to be an indicator of muscle tension in a given variant of the experiment.

Differences in a normalized EMG amplitude (NEMG) according to variants of the experimentwhich were differentiated according to parameters describing the experiment conditions (the level of the relative force of the handgrip and relative force of supporting upper limb posture)—were examined.

An analysis of the EMG signal was conducted by using software that was part of the ME4000 apparatus. For statistical analysis, Statistica software v. 6 (from StatSoft) was used. To establish differences between specific groups of variants the $t$ test was employed.

\section{RESULTS}

\subsection{Musculoskeletal Load Expressed by EMG}

Variants of the experiments were differentiated by two factors: the four Boundary Upper Limb Postures, which influenced the force of supporting the upper limb in the determined posture or upper limb movement, and the level of relative handgrip force exerted in the Boundary Upper Limb Postures. In other words it was differentiated by the level of the force of supporting the upper limb in a determined posture and by the level of handgrip force.

Figure 3 presents values of a normalized amplitude of the EMG signal (NEMG) for muscle trapezius pars descendents registered in each variant of the experiments. The EMG signal was normalized according to the EMG signal registered while performing Test 1 . The values of a normalized amplitude for the same Boundary Upper Limb Postures are very similar. There are no differences in to the level of handgrip force.

Figure 4 presents the EMG signal of the deltoideus pars anterior muscle normalized according to the maximum tension as exerted in Test 2. Thedeltoideus anteriormuscleis responsible for supporting the upper limb posture, which is well characterized by the NEMG parameter calculated for variants of the experiments. The influence of the upper limb posture on the activity of deltoideus pars anterior is described by the fact that there are differences in a normalized amplitude value in relation to Boundary Upper Limb Postures but there are no differences according to the level of relative handgrip force.

Figure 5 presents values of NEMG for the biceps brachii muscle. Normalization has been performed according to the EMG signal derived

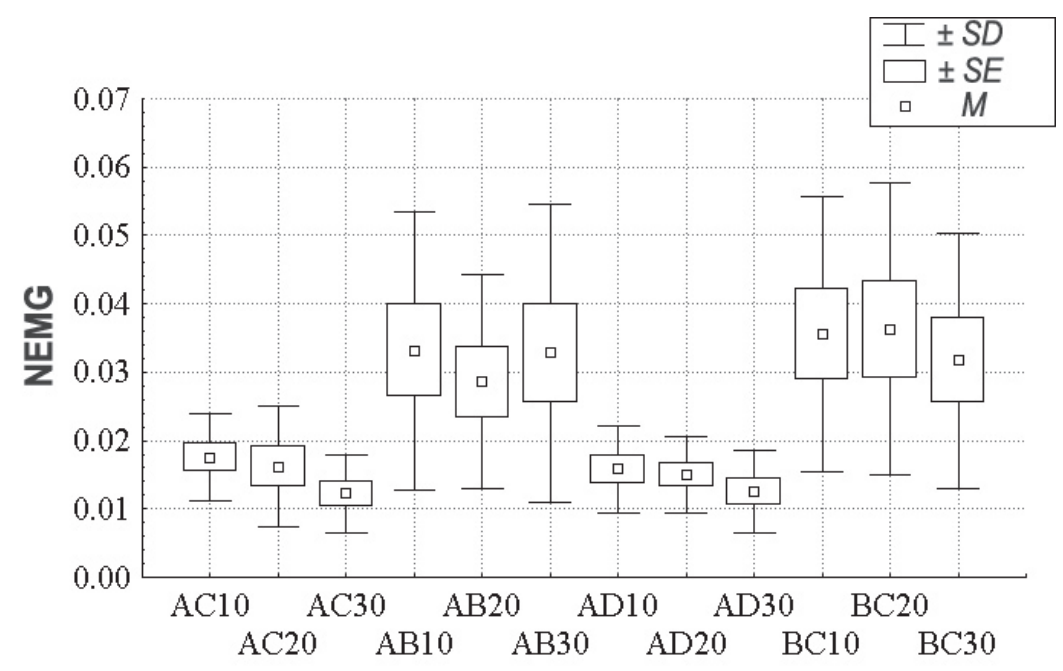

Figure 3. Values of a normalized electromyography amplitude (NEMG) in muscle trapezius descendent according to variants of the experiments (the letters correspond to Boundary Upper Limb Postures). 


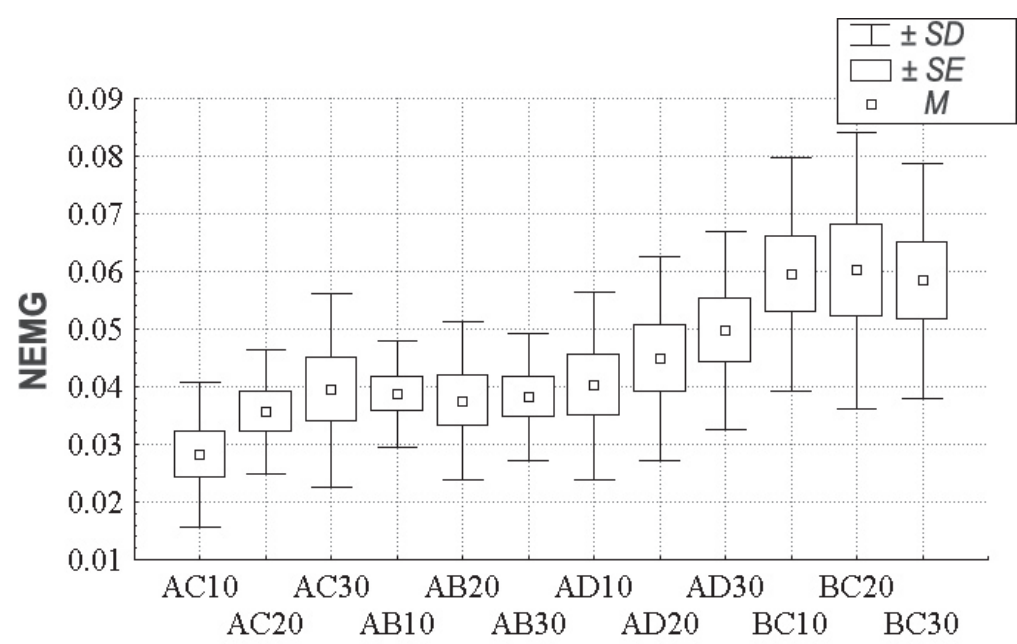

Figure 4. Values of a normalized electromyography amplitude (NEMG) in muscle deltoideus anterior according to variants of the experiments (the letters correspond to Boundary Upper Limb Postures).

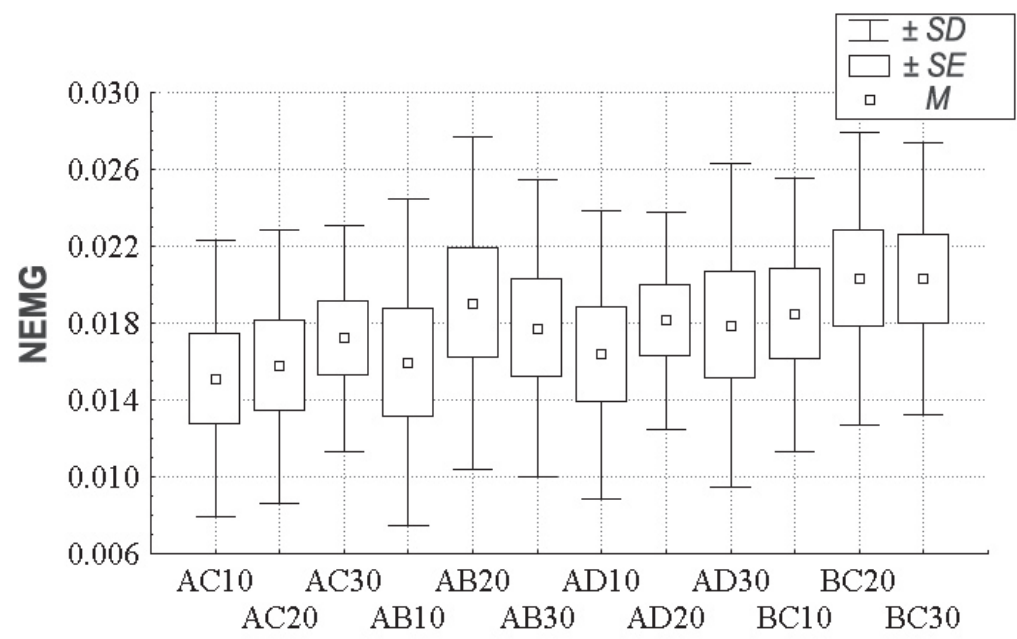

Figure 5. Values of a normalized electromyography amplitude (NEMG) in muscle biceps brachii according to variants of the experiments (the letters correspond to Boundary Upper Limb Postures).

during maximum tension of the muscle in the exertion force in Test 3. Values of the NEMG parameter are on a very similar low level (about $0.02)$ for all variants of the experiment. There are no differences either according to handgrip force level or to force of supporting the upper limb in the Boundary Upper Lomb Postures.

The amplitude of the EMG signal of the extensor carpi radialis and flexor carpi ulnaris muscles normalized to the signal obtained in Test 4 are presented in Figures 6 and 7 respectively. Both of the forearm muscles show similar tendencies in tension. It does not change according to the force of sustaining the upper limb in the determined posture. However, it differs very strongly according to the level of the relative handgrip force.
For each of the examined muscles a $t$ test for a group of variants comprising variants with the same Boundary Upper Limb Postures and different relative force levels was performed. It analyzed the differences in a normalized amplitude according to Boundary Upper Limb Postures. There were statistically significant differences only for muscles trapezius pars descendent and deltoideus pars anterior. For muscle trapezius there were statistically significant differences between the following group of variants: $\mathrm{AC}-\mathrm{AB}, \mathrm{AC}-\mathrm{AD}$, $\mathrm{AD}-\mathrm{AB}, \mathrm{BC}-\mathrm{AB}, \mathrm{BC}-\mathrm{AD}$ (the letters correspond to the Boundary Upper Limb Postures). For the deltoideus pars anterior muscle there were statistically significant differences between all the groups of variants comprising variants with the three levels of results for AC and AD. 


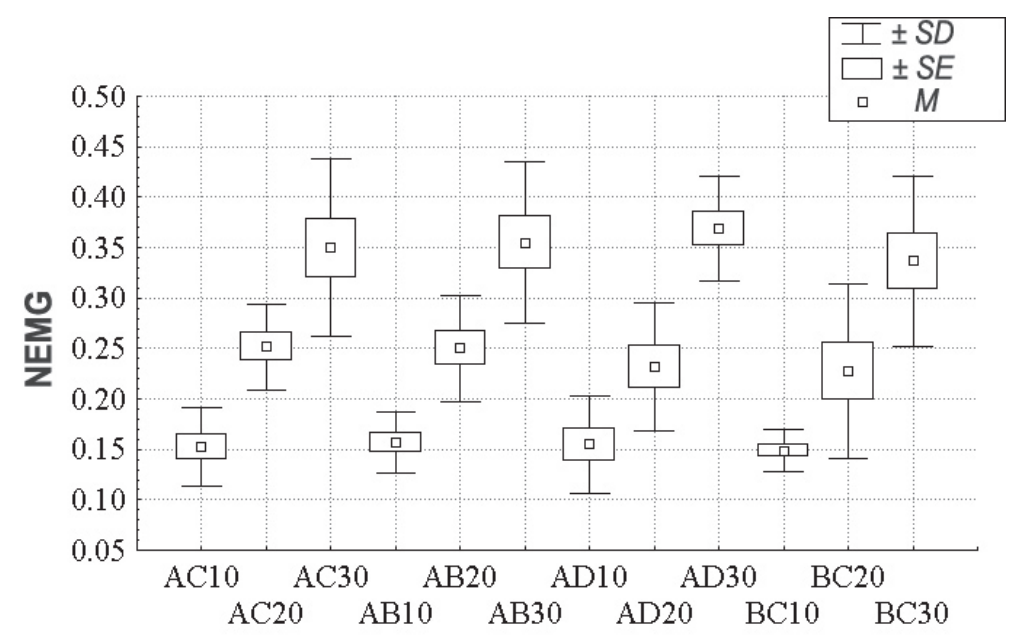

Figure 6. Values of a normalized electromyography amplitude (NEMG) in muscle extensor carpi radialis according to variants of the experiments (the letters correspond to Boundary Upper Limb Postures).

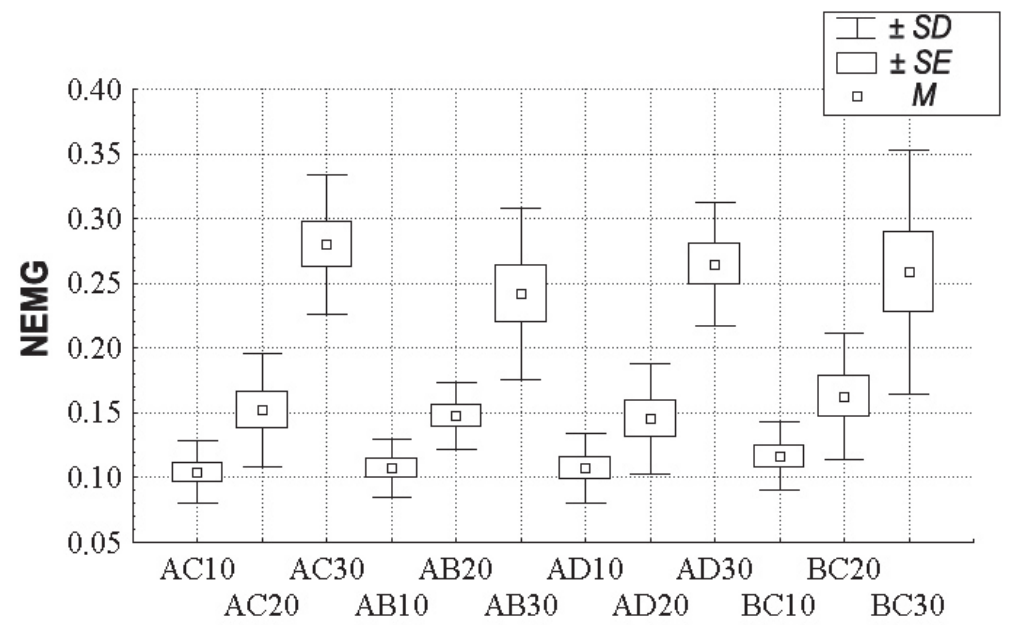

Figure 7. Values of a normalized electromyography amplitude (NEMG) in muscle flexor carpi ulnaris according to variants of the experiments (the letters correspond to Boundary Upper Limb Postures).

For comparison, calculations were done on the physiological indicator of musculoskeletal load. This indicator takes into account a normalized EMG amplitude of the shoulder, arm and forearm muscles. It is expressed as root mean square of a normalized amplitude (NEMG) of the five analyzed muscles. Such an indicator was calculated for each of the participants and each variant of external load. Mean values and standard deviation of the physiological indicator for each variant of the experiment are presented in Table 2.

\subsection{The Relationship Between $I C L$ and Physiological Indicator of Musculoskeletal Load}

Figure 8 presents values of the theoretical indicator of upper limb external load (ICL) and
TABLE 2. Mean Value and Standard Deviation of Root Mean Square of the Five Examined Muscles of Normalized Electromyography Amplitude (NEMG)

\begin{tabular}{lcc}
\hline Variant & $\boldsymbol{M}$ & $\boldsymbol{S D}$ \\
\hline AC10 & 0.090 & 0.0253 \\
AC20 & 0.135 & 0.0170 \\
AC30 & 0.205 & 0.0271 \\
AB10 & 0.095 & 0.0155 \\
AB20 & 0.132 & 0.0213 \\
AB30 & 0.195 & 0.0379 \\
AD10 & 0.089 & 0.0191 \\
AD20 & 0.128 & 0.0256 \\
AD30 & 0.206 & 0.0203 \\
BC10 & 0.085 & 0.0201 \\
BC20 & 0.132 & 0.0334 \\
BC30 & 0.195 & 0.0436 \\
\hline
\end{tabular}




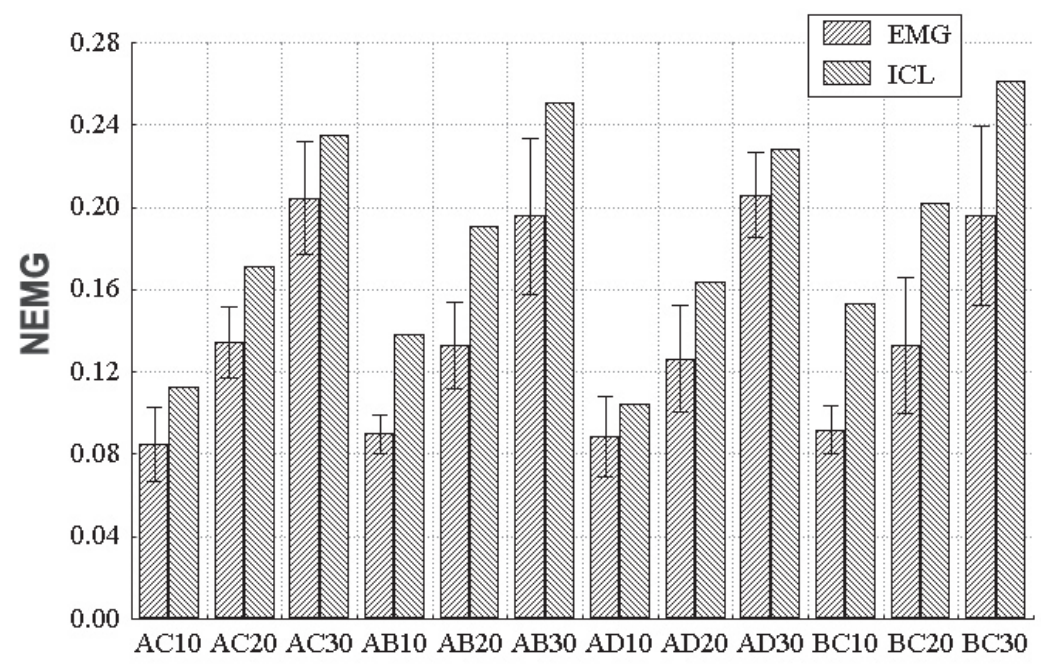

Figure 8. Mean values and standard deviation of the Integrated Cycle Load (ICL) and root mean square of the five examined muscles of a normalized electromyography amplitude (NEMG). Notes. EMG-electromyography.

the physiological indicator of musculoskeletal load of the upper limb assessed on the basis of EMG measurements for each of the variants of the experiment.

In the presented values for both parameters, similar tendencies according to the variants of the experiment are visible. There is strong correlation between values of those two indicators. The relationship between the theoretical indicator (ICL) and the physiological indicator (EMG) of upper musculoskeletal load is presented on Figure 9.

\section{DISCUSSION AND CONCLUSION}

The results of this study showed that while performing the experimental task, the forearm and hand muscles are mostly activated by handgrip force exertion as the arm and shoulder muscles are by the force of sustaining the weight of the upper limb, which changes according to upper limb posture. An analysis of a normalized EMG amplitude (NEMG) showed that the trapezius descendent and deltoideus anterior muscles were sensitive to changes in upper limb posture.

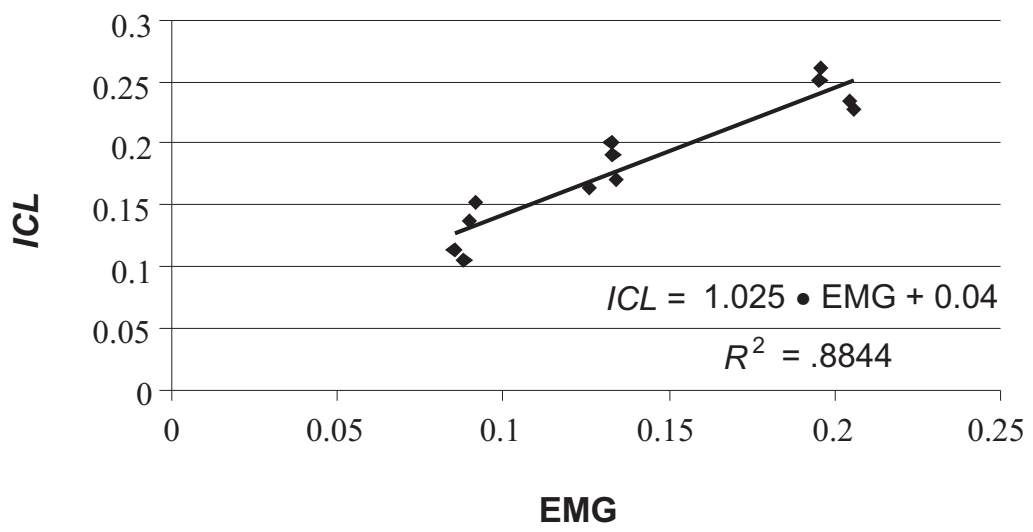

Figure 9. The relationship between the theoretical (Integrated Cycle Load, ICL) and physiological (root mean square of a normalized electromyography amplitude of the five examined muscles) indicator of musculoskeletal load. Notes. EMG-electromyography. 
Although the muscles of the forearm extensor, carpi radialis and flexor carpi ulnaris are not sensitive to upper limb posture, they are strongly influenced by the level of relative handgrip force. The obtained results are in step with the results of other studies, which confirmed that an amplitude of the EMG signal registered from the arm and shoulder muscles during a performed study can be a physiological indicator of musculoskeletal load [1, 2, 3, 4, 13, 19].

The extensor carpi radialis longus and flexor carpi ulnaris muscles are responsible for handgrip force exertion. Ohtsuki's [20] study showed correlation between handgrip force and the integrated amplitude of the EMG signal for finger flexor muscles. In the present study apart from those two forearm muscles, the trapezius and deltoideus muscles were also examined. The activities of those two muscles are mostly connected with supporting the weight of the upper limb and tension in those muscles changes according to the upper limb posture [21]. It is generally accepted that the role of the trapezius muscle is to support posture and that this muscle is responsible for upper limb posture [3, 22, 23, 24]. A similar role of the anterior deltoideus and middle deltoideus muscle has been found in the study of Giroux and Lamontagne [25] and Kronberg et al., [26].

$I C L$ expresses upper limb musculoskeletal load as a function of external parameters characterizing the performed repetitive task. The results of the study showed strong correlation between the theoretical indicator (ICL) and the physiological indicator (EMG). Although the values differ, what is meaningful is that there is correlation between those two indicators. Both of the indicators have no units (they are dimensionless). The values of the physiological indicator are lower than of $I C L$, which can be due to the fact that a limited number of muscles was taken into consideration.

Taking into consideration the fact that electromyography is a well established and universally approved measure of muscle tension, it can be accepted that the physiological indicator-root mean square of a normalized amplitude of the examined muscle-expresses muscular load. The strong correlation between $I C L$ and the physiological indicator of upper limb load proves that the developed indicator (ICL) can be a theoretical indicator of upper limb musculoskeletal load.

\section{REFERENCES}

1. Cook T, Rosencrance J, Zimmermann C, Gerleman D, Ludewig P. Electromyographic analysis of a repetitive hand gripping task. International Journal of Occupational Safety and Ergonomics (JOSE) 1998;4(2):185-200.

2. Dahalan JB, Fernandez JE. Psychophysical frequency for a gripping task. Int J Ind Ergon 1993;12:219-30.

3. Hagberg M. Work load and fatigue in repetitive arm elevations. Ergonomics 1981; 24(7):543-55.

4. Kim $\mathrm{CH}$, Fernandez JE. Psychophysical frequency for a gripping task. Int J Ind Ergon 1993;12:209-18.

5. Kobryn U, Hoffmann B, Küchler G. Effects of repeated fatiguing rythmical hand work. Eur J Appl Physiol 1981;47:271-97.

6. Marley R, Fernandez J. Psychophysical frequency and sustained exertion at varying wrist postures for a drilling task. Ergonomics 1995;38(2):303-25.

7. Roman-Liu D, Tokarski T, Kamińska J. Assessment of musculoskeletal load of trapezius and deltoid muscles during activity of hand. International Journal of Occupational Safety and Ergonomics (JOSE) 2001;7(2);179-93.

8. Bystrom SEG, Kilbom Å. Physiological response in the forearm during and after isometric intermittent handgrip. Eur J Appl Physiol 1990;60:457-66.

9. Mathiassen SE. The influence of exercise/ rest shedule on the physiological and psychophysical response to isometric shoulder-neck exercise. Eur J Appl Physiol 1993;67:528-39.

10. Anti S. Relationship between time means of external load and EMG amplitude in long term myoelectric studies. Electromyogr Clin Neurophysiol 1977;17:45-53.

11. Christensen $\mathrm{H}$, LoMonaco $\mathrm{M}$, Dahl $\mathrm{K}$, Fuglsang-Frederiksen A. Processing of 
electrical activity in human muscle during a gradual increase in force. Electroencephalogr Clin Neurophysiol 1984;58:230-39.

12. De Luca CJ. The use of surface electromyography in biomechanics. J Appl Biomech 1997;13:135-63.

13. Grant KA, Habes DJ, Putz-Anderson V. Psychophysical and EMG correlates of force exertion in manual work. Int J Ind Ergon 1994;13:31-9.

14. Lawrence JH, De Luca CJ. Myoelectric signal versus force relationship in different human muscles, J Appl Physiol 1983;54:1653-9.

15. Roman-Liu D. Upper limb load as a function of repetitive task parameters: part 1 - a model of upper limb load. International Journal of Occupational Safety and Ergonomics (JOSE) 2005;11(1):93-102.

16. Kelly BT, Kadrmas WR, Kirkendall DT, Speer KP. Optimal normalization tests for shoulder muscle activation: an electromyographic study. J Orthop Res 1996;14:647-53.

17. Nieminen H., Takala E.P., Vikari-Juntura E. Normalization of electromyogram in the neck-shoulder region. Eur J Appl Physiol 1993;67:199-207.

18. Mirka GA. The quantification of EMG normalization error. Ergonomics 1991; 34(3):343-52.

19. Kim JY, Chung MK, Park JS. Measurement of physical work capacity during arm and shoulder lifting at various shoulder flexion and ad/abduction angles. Human
Factors and Ergonomics in Manufacturing 2003;13(2):153-63.

20. Ohtsuki T. Decrease in grip strength induced by simultaneous bilateral exertion with reference to finger strength. Ergonomics 1981;24(1):37-48.

21. Roman-Liu D, Tokarski T. EMG arm and forearm muscle activities with regard to handgrip force in relation to upper limb location. Acta of Bioengineering and Biomechanics 2002;4(2):33-47.

22. Herberts $\mathrm{P}$, Kadefors $\mathrm{R}$, Broman $\mathrm{H}$. Arm positioning in manual tasks, An electromyographic study of localised muscle fatigue, Ergonomics 1980;23:655-65.

23. Mathiassen SE, WinkelJ.Electromyographic activity in the shoulder-neck region according to arm position and glenohumeral torque. Eur J Appl Physiol 1990;61:370-9.

24. Sigholm G, Herberts P, Almström C, Kadefors R. Electromyographic analysis of shoulder muscle load. J Orthop Res 1984;1:379-86.

25. Giroux B, Lamontagne M. Net shoulder joint moment and muscular activity during light weight-handling at different displacements and frequencies. Ergonomics 1992;35(4):385-403.

26. Kronberg M, Nemeth $G$, Broström L. Muscle activity and coordination in the normal shoulder-an electromyographic study. Clin Orthop 1990;257:76-85. 